\title{
Some Result of Stability and Spectra Properties on Semigroup of Linear Operator
}

\author{
Kamilu Rauf*, Akinola Yussuff Akinyele, Mfon Okon Etuk, Rafiu Obashola Zubair, \\ Moses Adebowale Aasa
}

Department of Mathematics, University of Ilorin, Ilorin, Nigeria

Email: ${ }^{\star}$ raufkml@gmail.com

How to cite this paper: Rauf, K., Akinyele, A.Y., Etuk, M.O., Zubair, R.O. and Aasa, M.A. (2019) Some Result of Stability and Spectra Properties on Semigroup of Linear Operator. Advances in Pure Mathematics, 9, 43-51.

https://doi.org/10.4236/apm.2019.91003

Received: October 17, 2018

Accepted: January 22, 2019

Published: January 25, 2019

Copyright $\odot 2019$ by author(s) and Scientific Research Publishing Inc. This work is licensed under the Creative Commons Attribution International License (CC BY 4.0). http://creativecommons.org/licenses/by/4.0/

\section{Open Access}

\begin{abstract}
This paper consists of some properties of a new subclass of semigroup of linear operator. The stability and spectra analysis of $\omega$-order preserving partial contraction mapping $\left(\omega-O C P_{n}\right)$ are obtained. The results show that operators on the proposed $\omega-O C P_{n}$ are densely defined and closed. Several existing results in the literature are contained in this work.
\end{abstract}

\section{Keywords}

Contraction Mapping, Semigroup, Banach Space, Resolvent and Bounded Operator

\section{Introduction}

The theory of stability is important since stability plays a central role in the structural theory of operators such as semigroup of linear operator, contraction semigroup, invariant subspace theory and to mention but few. The theory of stability is rich in which concerns the methods and ideas, and this shall be one of the main points of this paper. The recent advances deeply interact with modern topics from complex function theory, harmonic analysis, the geometry of Banach spaces, and spectra theory [1].

Another main focus of this paper is spectra analysis of a semigroup of linear operator, in which we use the resolvent to describe the relationship between the spectrum of $A$ and of the semigroup operator $(T(t))_{t \geq 0}$ and also determine the bounded linear operator $A$ as the generators of one-parameter semigroups. Resolvent operators are particularly useful in the analysis of Sturm-Liouville operators and several others operators both bounded and unbounded.

Let $X$ be a Banach space, $X_{n} \subseteq X$ be a finite set, $(T(t))_{t \geq 0}$ the $C_{0}$-semigroup which is strongly continuous one parameter semigroup of bounded linear oper- 
ator in $X, \omega$ - $O C P_{n}$ be $\omega$-order-preserving partial contraction mapping (semigroup of linear operator) which is an example of $C_{0}$-semigroup. Similarly, let $\operatorname{Mm}(\mathbb{N})$ be a matrix, $L(X)$ be a bounded linear operator on $X, P_{n}$ a partial transformation semigroup, $\rho(A)$ a resolvent set, $\sigma(A)$ be spectrum and $A$ is a generator of $C_{0}$-semigroup.

This paper will focus on results of stability and spectra analysis of $\omega-O C P_{n}$ on Banach space as an example of a semigroup of linear called $C_{0}$-semigroup, and thereby establish the relationship between a semigroup, its generator and the resolvent as in Figure 1.

In [2], Batty obtained some spectral conditions for stability of one-parameter semigroup and also revealed some asymptotic behaviour of semigroup of operator, see also, Batty et al. [3]. Chill and Tomilov [4] established some resolvent approach to stability operator semigroup. Räbiger and Wolf in [5] deduced some spectral and asymptotic properties of dominated operator. For relevant work on non-linear and one-parameter semigroups, see ([6] and [7]). The aim of this work is, therefore, to obtain stability and spectra analysis on a new subclass of semigroup of linear operator.

\section{Preliminaries}

The following definitions are crucial to the proof of our main results.

Definition 2.1: (Stable Semigroup [8])

A strongly continuous semigroup $(T(t))_{t \geq 0}$ is called

1) Uniformly exponentially stable if there exists $\epsilon>0$ such that

$$
\lim _{t \rightarrow \infty} e^{\epsilon t}\|T(t)\|=0
$$

2) Uniformly stable if

$$
\lim _{t \rightarrow \infty}\|T(t)\|=0
$$

3) Strongly stable if

$$
\lim _{t \rightarrow \infty}\left\|T(t)_{x}\right\|=0 \quad \forall x \in X .
$$

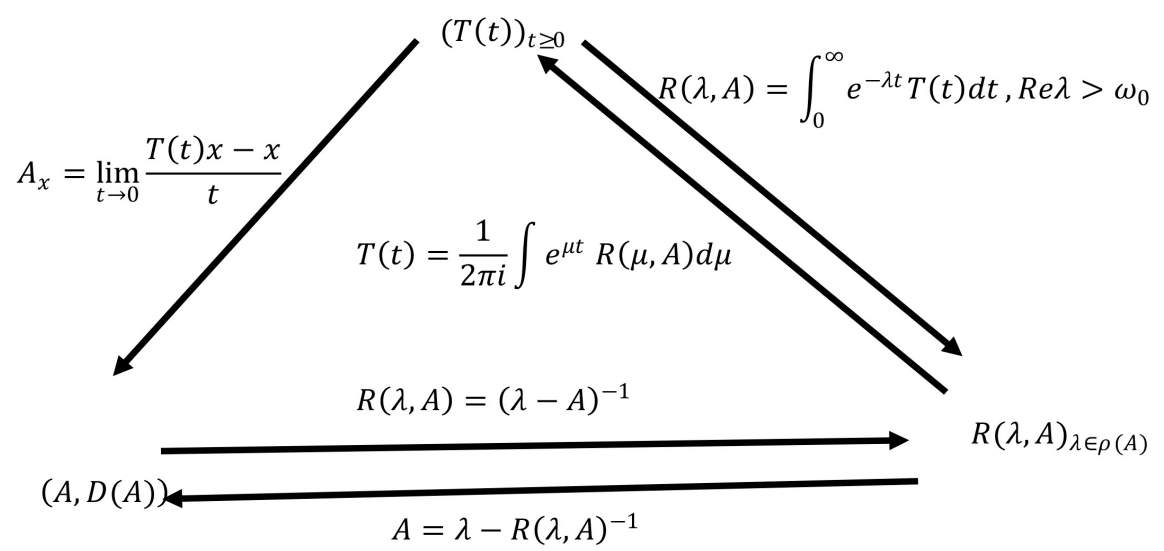

Figure 1. Diagrammatical representation of relationship between a semigroup, its generator and its resolvent [8]. 
Definition 2.2: ( $C_{0}$-Semigroup [9])

A $C_{0}$-Semigroup is a strongly continuous one parameter semigroup of bounded linear operator on Banach space.

Definition 2.3: $\left(\omega-O C P_{n}[10]\right)$

A transformation $\alpha \in P_{n}$ is called $\omega$-order-preserving partial contraction mapping if $\forall x, y \in \operatorname{Dom} \alpha: x \leq y \Rightarrow \alpha x \leq \alpha y$ and at least one of its transformation must satisfy $\alpha y=y$ such that $T(t+s)=T(t) T(s)$ whenever $t, s>0$ and otherwise for $T(0)=I$.

Definition 2.4: (Core [8])

Let $A$ be a closed linear operator with domain $D(A)$ and range $R(A)$ in a Banach space $X$. A subspace $D$ of $D(A)$ is called a core if $A$ is the closure of its restriction to $D$.

Definition 2.5: (Resolvent Set [11])

We define the resolvent set of $A$ denoted by $\rho(A)$ set of all $\lambda \in \mathbb{C}$ such that $\lambda I-A$ is one-to-one with range equal to $X$.

Definition 2.6: (Spectrum [11])

The spectrum of $A$ denoted by $\sigma(A)$ is defined as the complement of the resolvent set.

Definition 2.7: (Hyperbolic [12])

A semigroup $(T(t))_{t \geq 0}$ on a Banach space $X$ is called hyperbolic if $X$ can be written as direct sum $X=X_{s} \oplus X_{u}$ of two $(T(t))_{t \geq 0}$-invariant, closed subspaces $X_{s}, X_{u}$ such that the restricted semigroups $\left(T_{s}(t)\right)_{t \geq 0}$ on $X_{s}$ and $\left(T_{u}(t)\right)_{t \geq 0}$ on $X_{u}$ satisfy the following conditions:

1) The semigroup $\left(T_{s}(t)\right)_{t \geq 0}$ is uniformly exponentially stable on $X_{s}$.

2) The operator $T_{u}(t)$ are invertible on $X_{u}$, and $\left(T_{u}(t)^{-1}\right)_{t \geq 0}$ is uniformly exponentially stable on $X_{u}$.

\section{Some Basic Spectral Properties}

1) To any linear operator $A$ we associate its spectral bound defined by

$$
\operatorname{Spec}(A)=\operatorname{Sup}\{\operatorname{Re} \lambda: \lambda \in \sigma(A)\} .
$$

2) Resolvent set: $\rho(A)=\{\lambda \in \mathbb{C}: \lambda-A: D(A) \rightarrow X$ is bijective $\}$.

3) Spectrum: $\sigma(A)=\mathbb{C} / \rho(A)$.

4) Resolvent: $R(\lambda ; A)=(\lambda-A)^{-1} \forall \lambda \in \rho(A)$.

5) Resolvent equation: $R(\lambda ; A)-R(\mu ; A)=(\mu-\lambda) R(\lambda ; A) R(\mu ; A)$.

\section{Example 1:}

$2 \times 2$ matrix $\left[M_{m}\left(\mathbb{R}_{+}\right)\right]$

Suppose

$$
A=\left(\begin{array}{ll}
1 & 2 \\
2 & 2
\end{array}\right)
$$

and let $T(t)=e^{t A}$, then

$$
e^{t A}=\left(\begin{array}{cc}
e^{t} & e^{2 t} \\
e^{2 t} & e^{2 t}
\end{array}\right)
$$


$\underset{\text { Suppose }}{3 \times 3 \text { matrix }}\left[M_{m}\left(\mathbb{R}_{+}\right)\right]$

$$
A=\left(\begin{array}{lll}
1 & 2 & 3 \\
1 & 2 & 2 \\
- & 2 & 3
\end{array}\right)
$$

and let $T(t)=e^{t A}$, then

$$
e^{t A}=\left(\begin{array}{ccc}
e^{t} & e^{2 t} & e^{3 t} \\
e^{t} & e^{2 t} & e^{2 t} \\
I & e^{2 t} & e^{3 t}
\end{array}\right)
$$

\section{Example 2:}

$2 \times 2$ matrix $\left[M_{m}(\mathbb{C})\right]$, we have for each $\lambda>0$ such that $\lambda \in \rho(A)$ where $\rho(A)$ is a resolvent set on $X$.

Suppose we have

$$
A=\left(\begin{array}{ll}
1 & 2 \\
- & 2
\end{array}\right)
$$

and let $T(t)=e^{t A_{\lambda}}$, then

$$
e^{t A_{\lambda}}=\left(\begin{array}{cc}
e^{t \lambda} & e^{2 t \lambda} \\
I & e^{2 t \lambda}
\end{array}\right)
$$

\section{Example 3:}

Let $X=C_{u b}\left(\mathbb{R}_{+}\right)$be the space of all bounded and uniformly continuous function from $\mathbb{R}_{+}$to $\mathbb{R}$, endowed with the sup-norm $\|\cdot\|_{\infty}$ and let $\{T(t) ; t \geq 0\} \leq L(X)$ be defined by

$$
[T(t) f](s)=f(t+s)
$$

For each $f \in X$ and each $t, s \in \mathbb{R}_{+}$, one may easily verify that $\{T(t) ; t \geq 0\}$ satisfies the example 1 and 2 above.

\section{Main Results}

In this section, results of stability and spectral properties on $\omega-O C P_{n}$ in Banach space and on $C_{0}$-semigroup are considered:

\section{Theorem 3.1}

Suppose $X$ is a Banach space. Then a linear operator $A: D(A) \subseteq X \rightarrow X$ is an infinitesimal generator of a strongly continuous semigroup $(T(t))_{t \geq 0}$ on $X$ is uniformly exponentially stable if and only if for all $p \in[1, \infty)$ one has

$$
\int_{0}^{\infty}\|T(t) x\|^{p} \mathrm{~d} t<\infty
$$

for all $x \in X$ and $A \in \omega-O C P_{n}$.

Proof

If the semigroup is exponentially stable, then, the integral above is satisfied.

In order to show the converse implication, it suffices to verify that 


$$
\lim _{t \rightarrow \infty}\|T(t)\|=0 .
$$

So, we define for $n \in \mathbb{N}$, the operators $\mathfrak{I} \in\left(X, L^{p}\left(\mathbb{R}_{+}, X\right)\right)$ by

$$
\mathfrak{I}_{n} x=\phi_{[0, n]}(\cdot) T(\cdot)
$$

Then by assumption, the set $\left\{\mathfrak{\Im}_{n} x: n \in \mathbb{N}\right\} \subset L^{p}\left(\mathbb{R}_{+}, X\right)$ is bounded for each $x \in X$, hence by the uniform boundedness principle, there exists $C>0$ such that

$$
\int_{0}^{t}\|T(r) x\|^{p} \mathrm{~d} r \leq C^{p}\|x\|^{p} \text { for all } x \in X, t \geq 0 .
$$

On the other hand, there exist $M \geq 1$ and $w>0$ such that

$$
\|T(t)\| \leq M e^{w t} \text { for all } t \geq 0
$$

From the previous two inequalities, we obtain

$$
\begin{aligned}
\frac{1-e^{-p w t}}{p}\|T(t) x\|^{p} & =\int_{0}^{t} e^{-p w t}\|T(r) T(t-r) x\|^{p} \mathrm{~d} r \\
& \leq \int_{0}^{t} M^{p}\|T(t-r) x\|^{p} \mathrm{~d} r \\
& \leq M^{p} C^{p}\|x\|^{p} \quad \forall x \in X, t \geq 0 .
\end{aligned}
$$

Hence, there exists a constant $L>0$ such that

$$
\|T(t)\| \leq L \quad \forall t \geq 0
$$

Considering this, we conclude that

$$
\begin{aligned}
t\|T(t) x\|^{p} & =\int_{0}^{t}\|T(t-r) T(r) x\|^{p} \mathrm{~d} r \\
& \leq \int_{0}^{t} L^{p}\|T(r) x\|^{p} \mathrm{~d} r \\
& \leq L^{p} C^{p}\|x\|^{p} \quad \forall x \in X, t \geq 0
\end{aligned}
$$

and therefore

$$
\|T(t)\| \leq L C t^{\frac{-1}{p}} \quad \forall t>0 .
$$

This implies

$$
\lim _{t \rightarrow \infty}\|T(t)\|=0
$$

Hence the proof is complete.

\section{Proposition 3.2}

Suppose $X$ is a Banach space and $A: D(A) \subseteq X \rightarrow X$ where $A \in \omega-O C P_{n}$ is the infinitesimal generator for a strongly continuous semigroup $(T(t))_{t \geq 0}$, then the following assertions are equivalent.

1) $(T(t))_{t \geq 0}$ is hyperbolic.

2) $(T(t))_{t \geq 0} \cap \Gamma=\phi$ for all $t>0$.

\section{Proof}

The proof of implication 1) $\Rightarrow$ 2) starts from the observation that $\sigma(T(t))=\sigma\left(A_{s}\right) \cup \sigma\left(A_{u}\right)$ because of the direct sum decomposition. 
By assumption, $\left(T_{s}(t)\right)_{t \geq 0}$ is uniformly exponentially stable; hence $r\left(T_{s}(t)\right)<1$ for $t>0$, and therefore

$$
\sigma\left(T_{s}(t)\right) \cap \Gamma=\phi
$$

By the same argument, we obtain that $r\left(T_{s}(t)^{-1}\right)<1$. Suppose

$$
\sigma\left(T_{u}(t)\right)=\left\{\lambda^{-1}: \lambda \in \sigma(A)^{-1}\right\}
$$

we conclude that $|\lambda|>1$ for each $\lambda \in \sigma\left(A_{u}\right)$; hence $\sigma\left(T_{u}(t)\right) \cap \Gamma=\phi$.

To prove 2) $\Rightarrow 1$ ), we fix $s>0$ such that $\sigma(T(s)) \cap \Gamma=\phi$ and we use the existence at a spectral projection $P$ corresponding to the spectral set

$$
\sigma(T(s))=\{\lambda \in \sigma(A):|\lambda|<1\} .
$$

Then the space $X$ is the direct sum $X=X_{s} \oplus X_{u}$ of the $(T(t))_{t \geq 0}$-invariant subspaces $X_{s}=\operatorname{rg} P$ and $X_{u}=\operatorname{ker} P$, where $A_{s} \subseteq X_{s}$ and $A_{u} \subseteq X_{u}$. Then the restriction $T_{s}(t) \in L\left(X_{s}\right)$ of $\mathrm{T}(\mathrm{s})$ has spectrum

$$
\sigma\left(T_{s}(s)\right)=\{\lambda \in \sigma(A):|\lambda|<1\}
$$

hence, spectral radius $r\left(T_{s}(s)\right)<1$. It follows that the semigroup $\left(T_{s}(t)\right)_{t \geq 0}=(P T(t))_{t \geq 0}$ is uniformly exponentially stable on $X_{s}$.

Similarly, the restriction $T_{u}(s) \in L\left(X_{u}\right)$ of $T(s)$ in $X_{u}$ has spectrum

$$
\sigma\left(T_{u}(s)\right)=\{\lambda \in \sigma(A):|\lambda|>1\}
$$

hence is invertible on $X_{u}$. Clearly this implies that $T_{u}(t)$ is invertible for $0 \leq t \leq s$, while for $t>s$ we choose $n \in N$ such that $n s>t$. Then

$$
T_{u}(s)^{n}=T_{u}(n s)=T(n s-t) T_{u}(t)=T_{u}(t) T_{u}(n s-t)
$$

hence $T_{u}(t)$ is invertible, since $T_{u}(s)$ is bijective.

Moreover, for the spectral radius, we have $r\left(T_{u}^{-1}(s)\right)<1$, and again this implies uniformly exponentially stable for the semigroup $\left(T_{u}(t)^{-1}\right)_{t \geq 0}$. Hence the proof.

\section{Theorem 3.3}

Suppose $A \in \omega-O C P_{n}$ and $\omega-O C P_{n} \in L(X)$. Let $A: D(A) \subseteq X \rightarrow X$ be a linear operator which satisfies:

a) $A$ is densely defined and closed; and

b) $(0,+\infty) \subseteq \rho(A)$ and for each $\lambda>0$, we have

$$
\|R(\lambda, A)\|_{L(X)} \leq \frac{1}{\lambda} .
$$

Then:

1) $\lim _{\lambda \rightarrow \infty} \lambda R(\lambda, A) x=x$ for each $x \in X$,

2) $A_{\lambda} x=\lambda^{2} R(\lambda, A) x-\lambda x$ for each $x \in X$,

3) $\lim _{\lambda \rightarrow \infty} A_{\lambda} x=A x$ for each $x \in D(A)$ and,

4) $A_{\lambda}$ is the infinitesimal generator of a uniformly continuous semigroup $\left\{e^{t A \lambda} ; t \geq 0\right\}$ satisfying 


$$
\left\|e^{t A \lambda}\right\|_{L(X)} \leq 1
$$

for each $t \geq 0$. In addition for each $x \in X$ and $\lambda, \mu>0$, we have

$$
\left\|e^{t A \lambda} x-e^{t A \mu} x\right\| \leq t\left\|A_{\lambda} x-A_{\mu} x\right\| .
$$

\section{Proof}

Let $x \in D(A)$ and $\lambda>0$. Then we have

$$
\|\lambda R(\lambda, A) x-x\|=\|A R(\lambda, A) A x\| \leq \frac{1}{\lambda}\|A x\|
$$

and as a result

$$
\lim _{\lambda \rightarrow \infty} \lambda R(\lambda, A) x=x
$$

for each $x \in D(A)$.

Since $D(A)$ is dense in $X$ and

$$
\|\lambda R(\lambda, A)\|_{L(X)} \leq 1
$$

and from (3.12), we deduce

$$
\lim _{\lambda \rightarrow \infty} \lambda R(\lambda, A) x=x .
$$

To show 2). Let us remark that we have successively

$$
\lambda^{2} R(\lambda, A)-\lambda I=\lambda^{2} R(\lambda, A)-\lambda(\lambda I-A) R(\lambda, A)=\lambda A R(\lambda, A)=A_{\lambda}
$$

So, if $x \in D(A)$, by 1$)$, we have

$$
\lim _{\lambda \rightarrow \infty} A_{\lambda} x=\lim _{\lambda \rightarrow \infty} \lambda R(\lambda, A) x=\lim _{\lambda \rightarrow \infty} \lambda R(\lambda, A) A x=A x,
$$

which complete the proof of 2) and 3).

To show that $\left\|e^{t A \lambda}\right\|_{L(X)} \leq 1$ for each $t \geq 0$. Since $A_{\lambda} \in \omega-O C P_{n}$ and $\omega-O C P_{n} \in L(X)$, then by theorem of uniformly continuous semigroup, it follows that its generates a uniformly semigroup $\left\{e^{t A_{\lambda}} ; t \geq 0\right\}$.

In order to show that $\left\|e^{t A_{\lambda}}\right\|_{L(X)} \leq 1$, let us remark that, by virtue of $A_{\lambda} x=\lambda^{2} R(\lambda, A) x-\lambda x$ for each $x \in X$ and $\left.\mathrm{b}\right)$, we have

$$
\begin{aligned}
\left\|e^{t A_{\lambda}}\right\|_{L(X)} & =\left\|e^{t \lambda^{2} R(\lambda, A)-t \lambda I}\right\|_{L(X)} \leq\left\|e^{t \lambda^{2} R(\lambda, A)}\right\|_{L(X)}\left\|e^{-t \lambda I}\right\|_{L(X)} \\
& \leq e^{t \lambda^{2}\|R(\lambda, A)\|_{L(X)}} e^{-t \lambda I} \leq e^{t \lambda I} e^{-t \lambda I}=1
\end{aligned}
$$

Since $A_{\lambda}, A_{\mu}, e^{t A_{\lambda}}$ and $e^{t A_{\mu}}$ commute each to another for each $\lambda, \mu \in \rho(A)$ and $\lambda, \mu>0$, we have

$$
\begin{aligned}
\left\|e^{t A_{\lambda}} x-e^{t A_{\mu}} x\right\| & =\left\|\int_{0}^{1} \frac{\mathrm{d}}{\mathrm{d} s}\left(e^{s t A_{\lambda}} e^{(1-s) t A_{\mu}} x\right) \mathrm{d} s\right\| \\
& \leq \int_{0}^{1} t\left\|e^{s t A_{\lambda}} e^{(1-s) t A_{\mu}}\left(A_{\lambda} x-A_{\mu} x\right)\right\| \mathrm{d} s \\
& \leq t\left\|A_{\lambda} x-A_{\mu} x\right\|
\end{aligned}
$$

Hence the proof is complete.

Theorem 3.4

For $A \in \omega$-OCP $P_{n}$, we have $A: D(A) \subseteq X \rightarrow X$ to be a linear operator satis- 
fying both $(0,+\infty) \subseteq \sigma(A)$ and

$$
\left\|\lambda^{n} R(\lambda, A)^{n}\right\|_{L(X)} \leq M
$$

for each $n \in N$ and $\lambda>0$ and if $\lambda, \mu$ are regular values, i.e. $\lambda, \mu \in \rho(A)$ and $R(\lambda, A), R(\mu, A) \in L(X)$, then there exist:

1) $R(\lambda, A)-R(\mu, A)=(\mu-\lambda) R(\lambda, A) R(\mu, A)$.

2) $\|x\| \leq|x| \leq M\|x\|$.

3) $|\lambda R(\lambda, A) x| \leq|x|$ for each $x \in X$ and $\lambda>0$.

\section{Proof}

To prove 1), let us observe that

$$
\begin{aligned}
R(\lambda, A) & =R(\lambda, A)(\mu I-A) R(\mu, A) \\
& =R(\lambda, A)\{(\mu-\lambda) I+(\lambda I-A)\} R(\mu, A) \\
& =(\mu-\lambda) R(\lambda, A) R(\mu, A)+R(\mu, A)
\end{aligned}
$$

so that

$$
R(\lambda, A)-R(\mu, A)=(\mu-\lambda) R(\lambda, A) R(\mu, A)
$$

and this complete the proof of 1 ).

To prove 2), we assume for $\mu>0$ and let us define $|\cdot|_{\mu}: D(A) \subseteq X \rightarrow \mathbb{R}_{+}$ by

$$
|x|_{\mu}=\operatorname{Sup}_{n \in \mathbb{N}}\left\|\mu^{n} R(\mu, A)^{n} x\right\|,
$$

it's obvious that

$$
\|x\| \leq|x|_{\mu} \leq M\|x\|
$$

and

$$
|\mu R(\mu, A) x|_{\mu} \leq|x|_{\mu} .
$$

We want to prove that

$$
|\lambda R(\lambda, A) x|_{\mu} \leq|x|_{\mu}
$$

for each $\lambda \in(0, \mu]$.

So by resolvent Equation (3.17), we have

$$
R(\lambda, A) x=R(\mu, A)(x+(\mu-\lambda) R(\mu, A) x)
$$

and therefore

$$
|R(\lambda, A) x|_{\mu} \leq \frac{1}{\mu}|x|_{\mu}+\left(1-\frac{\lambda}{\mu}\right)|R(\lambda, A) x|_{\mu} .
$$

Consequently $\lambda|R(\lambda, A) x|_{\mu} \leq|x|_{\mu}$ which proves (3.20).

From (3.19) and (3.20), we deduced that, for each $n \in \mathbb{N}$ and $\lambda \in(0, \mu]$, we have

$$
\left\|\lambda^{n} R(\lambda, A)^{n} x\right\| \leq\left|\lambda^{n} R(\lambda, A)^{n} x\right| \leq|x|_{\mu}
$$

Passing to the Sup for $n \in \mathbb{N}$ on the left hand side of the inequality above, we 
now get $|x|_{\lambda} \leq|x|_{\mu}$ for each $\lambda \in(0, \mu]$. We can now define

$$
|x|=\lim _{\mu \rightarrow 0}|x|_{\mu}
$$

Since 2) readily follows from (3.19), and 3) from (3.21) by taking $n=1$, we have

$$
|\lambda R(\lambda, A) x| \leq|x|
$$

Hence the proof.

\section{Conflicts of Interest}

The authors declare no conflicts of interest regarding the publication of this paper.

\section{References}

[1] Banach, S. (1922) Surles Operation Dam Les Eusembles Abstracts et lear Application Aus Equation Integrals. Fundamenta Mathematicae, 3, 133-181. https://doi.org/10.4064/fm-3-1-133-181

[2] Batty, C.J.K. (1996) Spectral Condition for Stabilty of One-Parameter Semigroup. Journal of Differential Equations, 127, 87-96. https://doi.org/10.1006/jdeq.1996.0062

[3] Batty, C.J.K., Chill, R. and Tomilov, Y. (2002) Strong Stability of Bounded Evolution Families and Semigroup. Journal of Functional Analysis, 193, 116-139. https://doi.org/10.1006/jfan.2001.3917

[4] Chill, R. and Tomilov, Y. (2007) Stability Operator Semigroup. Banach Center Publication 75, Polish Academy of Sciences, Warsaw, 71-73.

[5] Räbiger, F. and Wolf, M.P.H. (2000) Spectral and Asymptotic Properties of Resolvent Dominated-Operators. Journal of the Australian Mathematical Society Series $A, 68,181-201$. https://doi.org/10.1017/S1446788700001944

[6] Ambrozie, C. and Müller, V. (2004) Invariant Subspaces for Polynomially Bounded Operators. Journal of Functional Analysis, 213, 321-345. https://doi.org/10.1016/j.jfa.2003.12.004

[7] Balakrishnan, A.V. (1960) Fractional Powers of Closed Operators and Semigroups Generated by Them. Pacific Journal of Mathematics, 10, 419-437. https://doi.org/10.2140/pjm.1960.10.419

[8] Ioan, I.V. (2003) $\mathrm{C}_{0}$-Semigroups and Applications. Mathematics Studies, 191, Elservier, North-Holland.

[9] Engel, K. and Nagel, R. (1999) One-Parameter Semigroups for Linear Equations. Graduate Texts in Mathematics, 194, Springer, New York.

[10] Rauf, K. and Akinyele, A.Y. (2019) Properties of $\omega$-Order-Preserving Partial Contraction Mapping and Its Relation to $\mathrm{C}_{0}$-Semigroup. International Journal of Mathematics and Computer Science, 14, 61-68.

[11] Bellem-Morante, A. and McBride, A. (1998) Applied Non-Linear Semigroups. Mathematics Methods in Practices. John Wiley and Sons, Chichester.

[12] Brezis, H. (2011) Functional Analysis, Sobolev Space and Partial Differential Equations. Springer, Berlin. 